Please send trade news information and illustrations to Arveen Bajaj at the BDJ, 64 Wimpole Street, London W1G 8YS. Trade news is supplied as a service to the reader and does not imply endorsement by the BDJ. Normal and prudent research should be exercised before purchase of use of any product mentioned.

\section{TRADE NEWS}

WHAT'S NEW

\section{Healthcare clothing}

The Klinidrape range manufactured by Molnlycke Health Care includes single use scrub suits with a V-neck slip over style tunic and trousers designed to give both men and women an attractive, comfortable fit. They are available in packs of 30 in small, medium, large and extra large sizes. It also includes medium, large and extra large disposable sterile gowns.

Other components of the range include Klinidrape caps in a variety of styles, Klinidrape Masks with earloops or tiebands and a wide selection of plain, split and aperture drapes and covers.

Reader response number 50

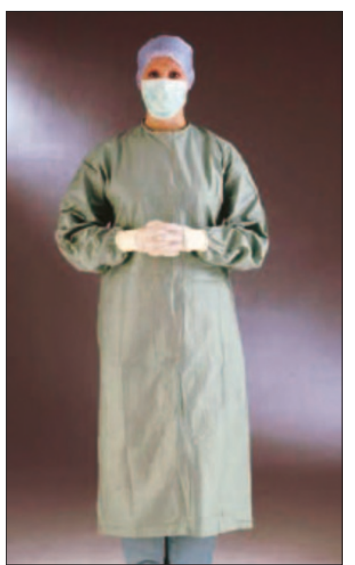

\section{Flexible comfort}

Fully adjustable Support Stools are designed to hold the occupant in the correct anatomical posture, its manufacturer claims.

Optional accessories include Hydros armrests which allow flexibility of move-

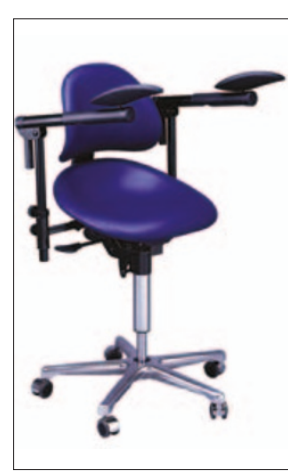
ment for the hands whilst supporting the arms. Others include Relax armrests, which hold the operator's mirror hand in place whilst also allowing freedom of movement and support.

Reader response number 51

\title{
Implantology unit special offer
}

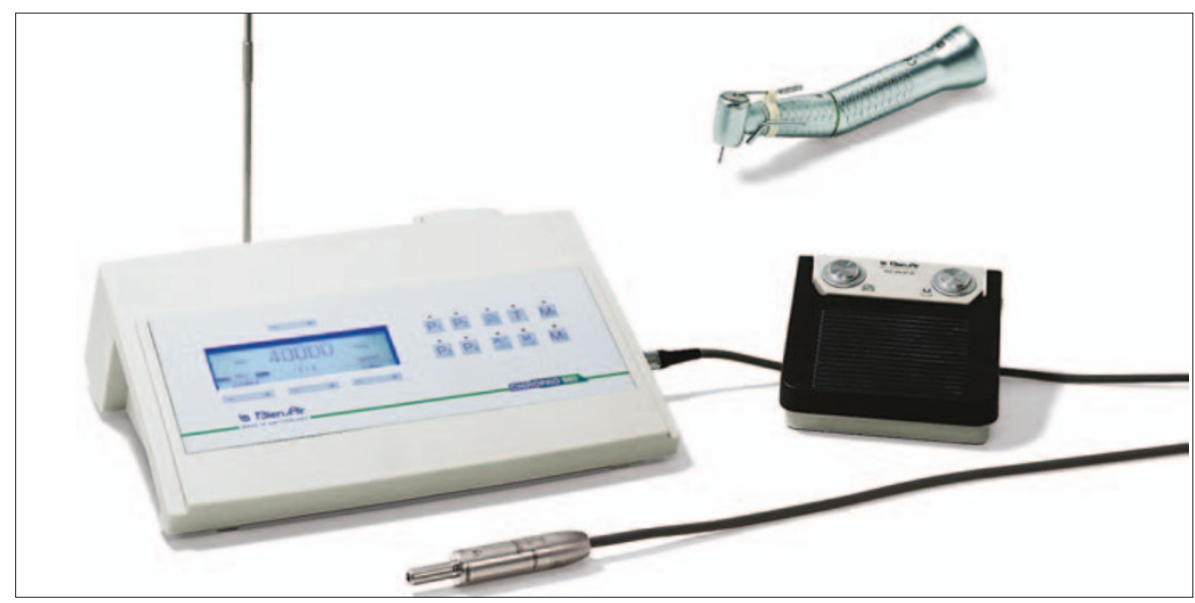

General Medical are currently offering the Chiropro 980 Implantology unit, from Bien Air, with a choice of either VAT free or free 30:1 contra-angled handpiece special offers. The Chiropro 980 Implantology unit is a table-top system for use with one or two Basch brushless, sterilisable micromotors. Each of its 8 programs can be manually adjusted, even if it is currently in use. It also offers a choice of 31 reduction or multiplication ratios, from 1000:1 to 1:6, and an integral peristaltic pump with a flow rate electronically controlled from 30 to $130 \mathrm{ml} / \mathrm{min}$. The Chiropro 980 system meets all the current EU safety standards.

It is supplied complete as an electronic table-top control unit with integrated peristaltic pump, two button foot control and a single Basch motor. Designed for use with Bien Air's 1:1, 30:1 or 100:1 speed reducing handpieces, the Chiropro 980's brushless motor is supplied with a $3 \mathrm{~m}$ cable and can be sterilised up to $136^{\circ} \mathrm{C}$.

Reader response number 52

\section{Relief for dry mouths}

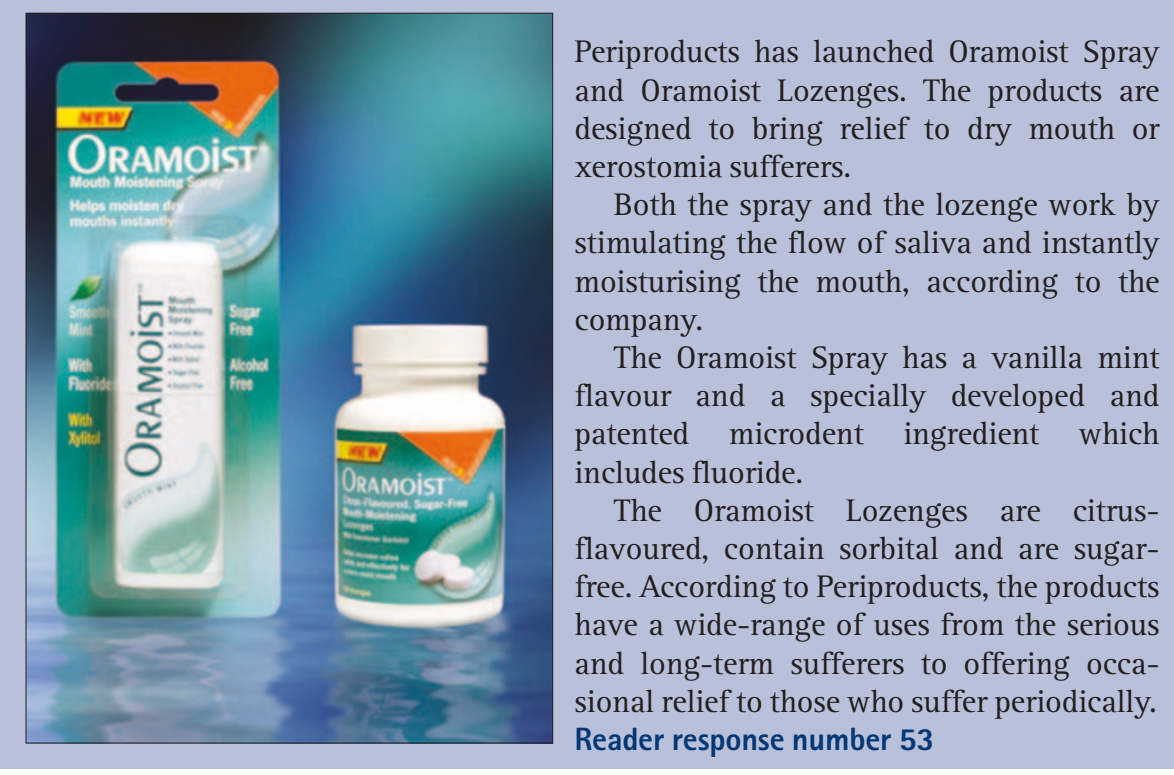




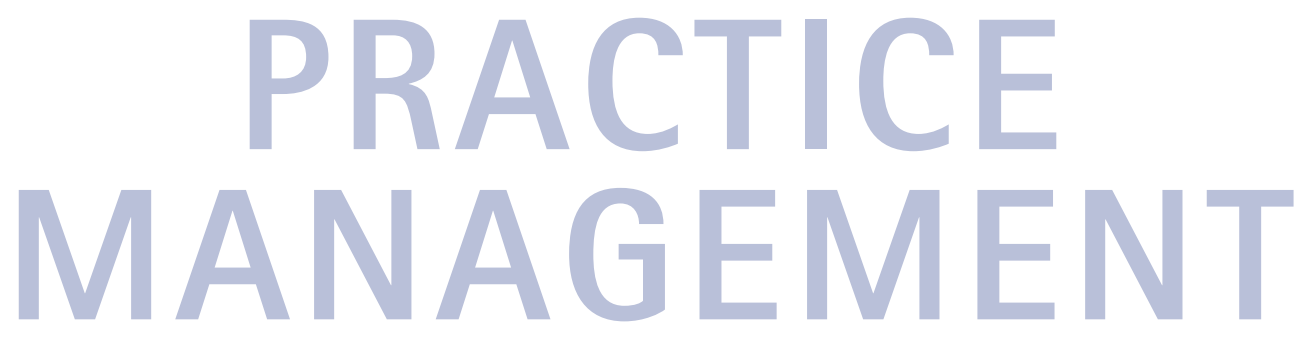

\section{Software solutions}

Sidexis is a software management system for intraoral and panoral digital radiograph images that is fully compatible with all mainstream practice management software packages on the market.

Because of its modular nature the system can be initially acquired as a standalone and integrated into a network at a later date. It works as part of a seamless network with a single database enabling $\mathrm{x}$-ray images and all other patient data to be available from anywhere within the practice. It is part of a product portfolio from Sirona that is handled by specialist dealers Sident Dental Systems. Reader response number 54

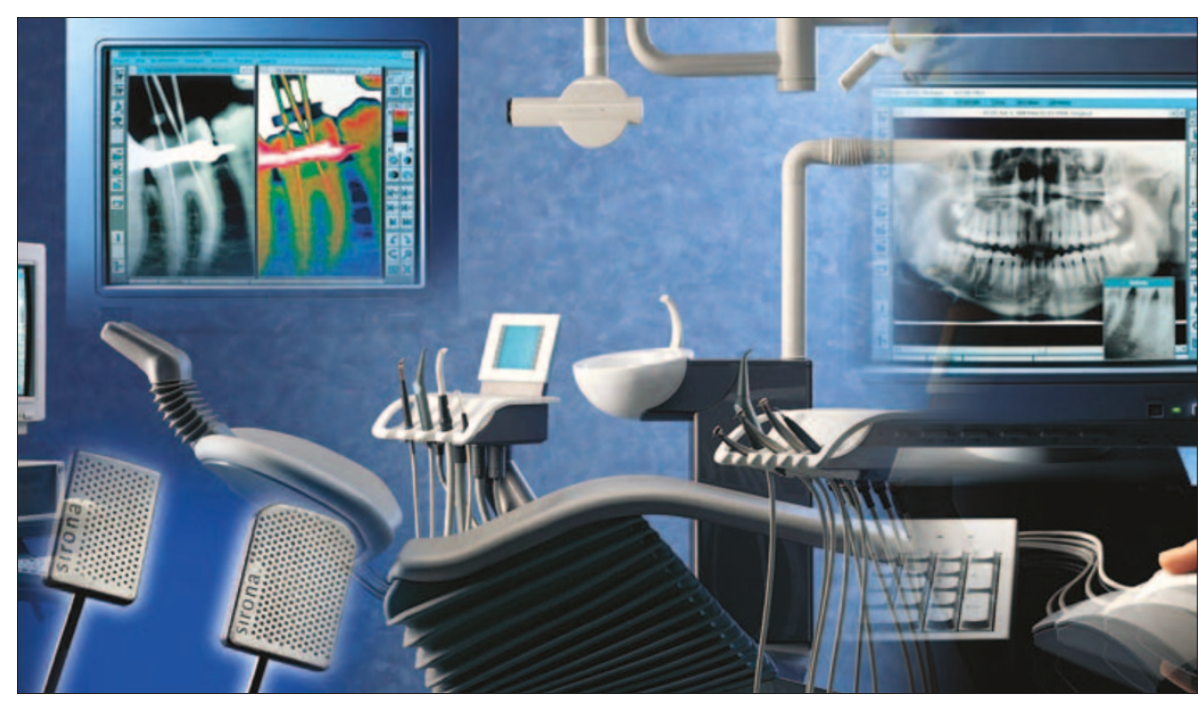




\section{Making surgeries a welcome place}

The Admor Practice Interiors range is designed to make waiting rooms and surgeries a more friendly environment for patients and staff. The new products include filing and storage, dental signage, reception posters, wall-fixed poster display systems and children's furniture. The range incorporates contemporary designs with wipe-clean surfaces to help keep it looking new and is available from the Admor catalogue. Admor are suppliers of administrative and customer service related products. For further information visit www.admor.co.uk.

Reader response number 55

\section{Bespoke storage}

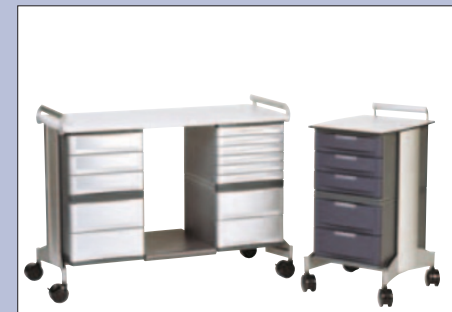

Highline bespoke healthcare storage solutions available from Support Stool are composed of modules, which contain sets of four different drawer sizes and can be combined in one of nine models to create units of the required height, width and mixture of drawer sizes to suit the individual. They are constructed from a combination of aluminium, steel and high quality plastics, which are resistant to most stains including blood.

Reader response number 56

\section{Practical style}

Henry Schein have developed a range of cabinetry solutions to suit different practices.

The Praxis range has been developed to provide the dentist with a budget option while the Pacific range reflects attention to detail and incorporates hardwood (mahogany) dovetailed drawers with concealed self-closing runners.

Finally the Penstock Range, with over 500 different colour options, has been designed with flexibility in mind to meet the needs of dentists who want a wider choice when selecting cabinetry.

Reader response number 57

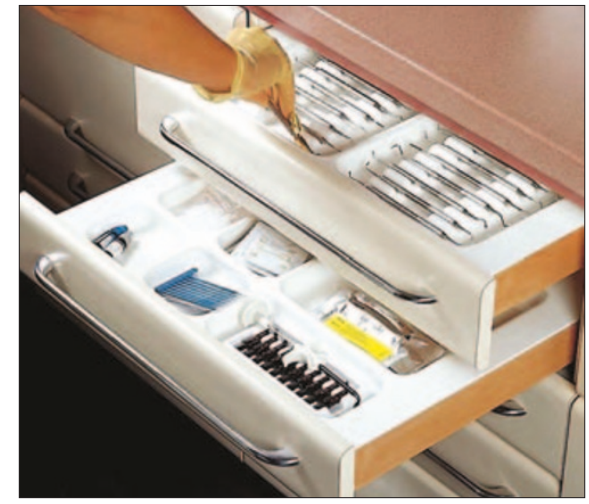




\section{Surgery planning}

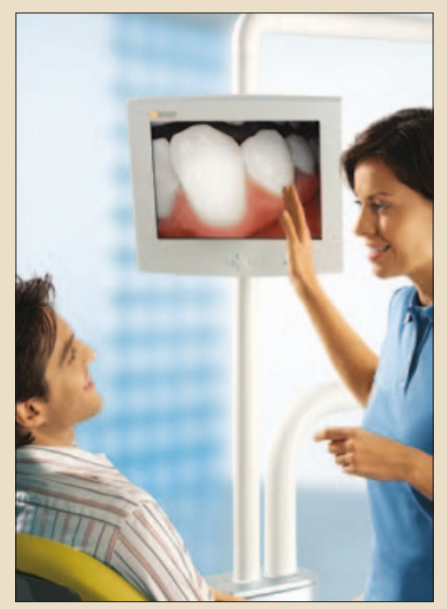

Sirona Dental Systems of Germany are able to provide bespoke surgery planning solutions for practices. The company's three general dealers in the mainland UK can work with practices at every stage of a refurbishment/re-equipping process. With an integrated product portfolio bespoke solutions can be realised from one manufacturer.

Reader response number 58

\section{Speed and accuracy}

The R4 Practice Management System from PracticeWorks includes Pathfinder, the only recognised address-finding software available in the UK. The programme was designed to save businesses valuable time by quickly and accurately displaying customers' addresses.

Pathfinder integrates easily into R4's patient records and is simple and quick to use. By typing in a patient's postcode it immediately presents the patient's street address on the screen. To complete the address the house name or number needs to be added, which can save time and keeps records accurate and updated.

Reader response number 59

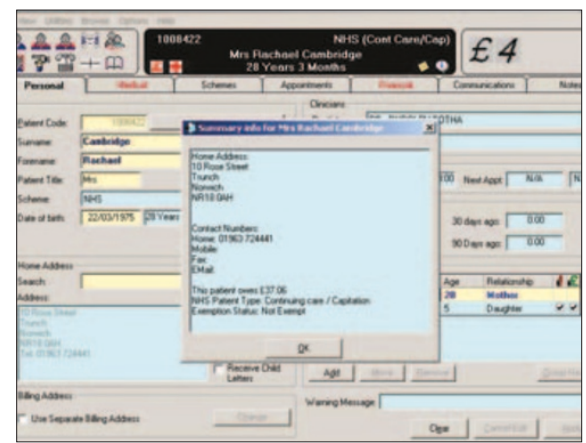

\section{Modern solutions for technology}

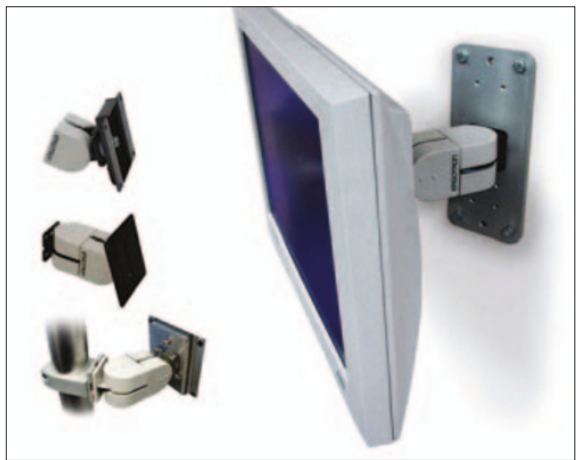

Henry Schein offer modern space saving solutions with Ergotron mounting arms. These enable dental technology items such as flat screens, keyboards and even CPUs to be safely and ergonomically located almost anywhere in the surgery.

Ergotron Mounting Solutions have many defining features including sleek modern design, robust construction, ergonomic surgery layout, is space saving and can be can be retro fitted.

Reader response number 60

\section{Streamlining analysis}

Orthometric, a cepholametric analysis programme available through the Dental Directory can help simplify and streamline several of the key treatment stages, according to its manufacturers. Patient profiles and landmark points can be calculated automatically, via the scanning of a radiograph. Orthometric allows a variety of images to be viewed, in either two or three dimensional format.

Following an appointment, Orthometric's report writing facility will allow you to make notes on treatment and save the information for future use. An added advantage is that the package is fully compatible with all Windows software.

Reader response number 61

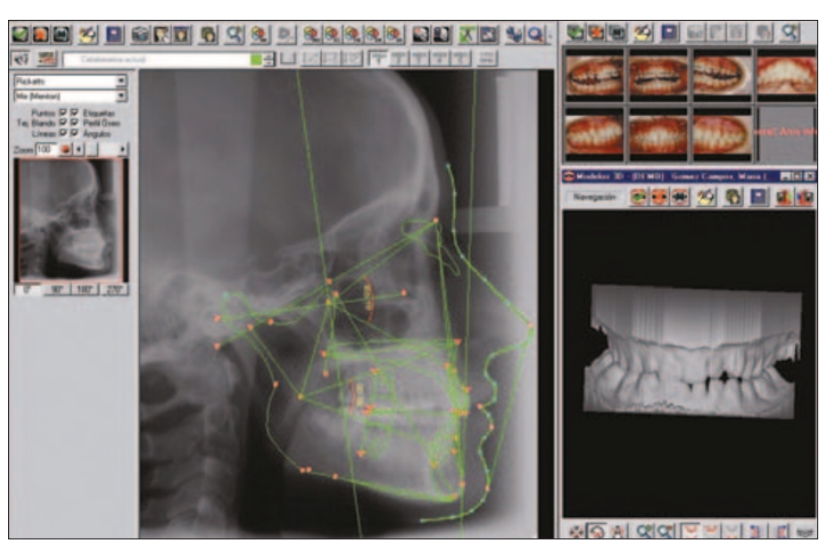

